\title{
The medical genetics of dystrophinopathies: Molecular genetic diagnosis and its impact on clinical practice
}

\author{
Alessandra Ferlini*, Marcella Neri, Francesca Gualandi \\ Section of Medical Genetics, Department of Medical Sciences, University of Ferrara, Italy
}

Received 6 April 2012; received in revised form 23 August 2012; accepted 4 September 2012

\begin{abstract}
A large variety of mutations in the dystrophin gene cause Duchenne and Becker muscular dystrophies, diseases affecting predominantly the striated muscles (skeletal and cardiac). Rare mutations also account for the allelic disorder isolated X-linked dilated cardiomyopathy. Dystrophin protein is encoded by a huge gene located on the $\mathrm{X}$ chromosome and the understanding of its complex genomic architecture has unraveled general key functions in gene expression regulation. Dystrophin also exists as a number of other tissue specific isoforms, some exclusively or predominantly expressed in the brain and/or in other tissues. Genotype definition of the dystrophin gene in patients with dystrophinopathies has taught us much about functionally important domains of the protein itself and has also provided insights regarding several regulatory mechanisms governing the gene expression profile. This review focuses on the current understanding of the dystrophin mutations heterogeneity, genotype-phenotype correlations, as well as interpretation of the functional significance of mutations that often require non routine genetic studies. It also explores the impact of genetic diagnosis on clinical definition and on the discovery of biomarkers and personalized therapies.

Our aim is to offer an overview of the medical genetic approach on the dystrophin gene and dystrophinopathies with implications for clinical practice and therapeutic perspectives.
\end{abstract}

(C) 2012 Elsevier B.V. All rights reserved.

Keywords: Duchenne and Becker muscular dystrophies; Dystrophin; Molecular genetics; Mutations; Genotype phenotype correlation

\section{The dystrophin gene and protein: structure, architecture and regulation}

The dystrophin gene was the first gene isolated by positional cloning and has proved to be the most complex genetic locus still identified.

Dystrophin and titin are the largest disease genes in the human genome [1,2]. Dystrophin spans 2.2 megabases at $\mathrm{Xp} 21$ on the human $\mathrm{X}$ chromosome and it is composed by 79 constitutive exons. Exon 79 is the longest $(2.7 \mathrm{~kb})$, being the one containing the full $3^{\prime}$ UTR region. The gene is driven by three main promoters, the upstream and

\footnotetext{
* Corresponding author. Address: Section of Medical Genetics, Department of Medical Sciences, University of Ferrara, Via Fossato di Mortara, 74, 44121 Ferrara, Italy. Tel.: +39 0532 974439; fax: +390532974406.

E-mail address: fla@unife.it (A. Ferlini).
}

ancient Brain (B), the Muscular (M) and the Purkinje (P), which guides the full length dystrophin transcription in a tissue/development specific way $[3,4]$. Furthermore, at least other four first exons have been recognized as adjacent to promoters driving short dystrophin isoforms, and localized within introns 29 (retinal isoforms or Dp260, R), 44 (Brain specific isoform or Dp140, B3), 55 (Schwann cells isoform or Dp116, S) and 62 (General isoform or Dp71, G) [1].

Several alternatively utilized exons, always located within introns, have also been described [1].

The architecture of the dystrophin gene, reporting the exon composition, isoforms, relevant domains and structural organization is reported in Fig. 1.

The DMD Leiden pages (http://www.dmd.nl/) report all the known intronic sequences and their relative accession numbers in the HGMP GeneBank. 


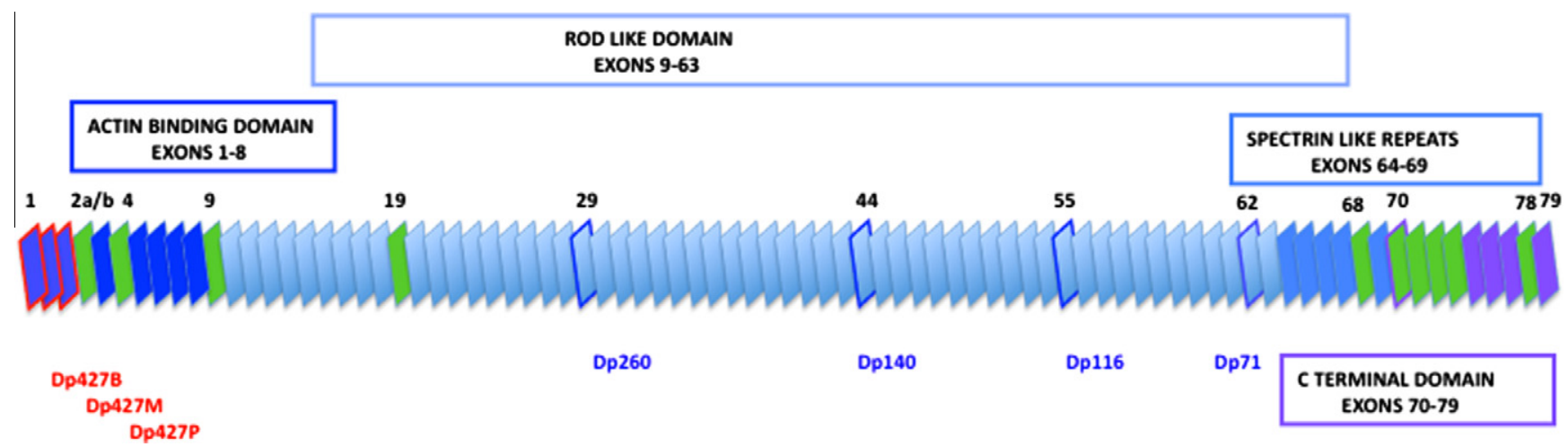

Fig. 1. Dystrophin gene structure and protein domains. Schematic representation of 79 exons of dystrophin gene with isoforms and protein domains. Lines in red represent the $5^{\prime}$ full length promoters and their first exon (isoforms Dp427B-M-P). Lines in blue represent the $3^{\prime}$ promoters and their first exons of isoforms: Dp260 (retinal), Dp140 (brain 3), Dp116 (Schwann cells), Dp71 (general). In green are represented exon alternatively spliced or skipped. Boxes' different blue/violet colors explain the protein domains corresponding to the different exonic regions.

The full sequencing of dystrophin introns has revealed that very large and in some cases huge introns are very common in the architecture of this gene. This is particularly true for the introns close to alternative transcription starting sites (as introns 1-Muscle, intron 1-Brain and intron 1-Purkinje, as well as intron 44). This fact together with the high occurrence of alternative splicing events in this gene raised the question why such large introns have been maintained during evolution. The dystrophin gene's unusually large intron size has been claimed as one of the major causes of the high mutation rate known to occur in some regions of the gene which give rise to two well known mutation hot spots $[1,5,6]$. Furthermore, the detailed analysis of the large introns, emerging from the full Genome Project sequencing, have revealed interesting characteristics and focused attention on possible roles and functions they may play in gene regulation, especially transcription and splicing $[7,8]$. Among dystrophin gene introns, the introns 7 and 44, which experience the highest recombination rates and are known to be mutational hot spots, seem to be sites of positive directional selection [9]. This suggests that these two introns may contain relevant regulatory motifs. Intron 7 is adjacent to a region of "exceptions to the rule" of the Monaco openreading-frame theory [10] (exons 3-7); in fact a restarting dystrophin ATG located within exon 6 has been postulated as mechanism to rescue dystrophin translation in mutations located within exon 2-6 [11]. These mutations cause a BMD phenotype despite of being out-of-frame, pointing out again that this region might be involved in regulatory processes. Introns are the segments involved in the DNA duplication process and duplication forks formation, fact that is mechanistically linked to the gene disruption due to non-allelic homologous recombination (NAHR), nonhomologous end joining (NHEJ), and microhomologymediated replication-dependent recombination (MMRDR) mechanisms known to explain DNA rearrangements associated with genomic disorders. Recently, Ankala et al., [12] have shown that non-recurrent "de novo" rearrangements within the dystrophin gene are associated with microhomologies or short insertions within introns. In many of the DMD cases studied the sequences upstream of the deletion breakpoints were repeatedly replicated. This suggests that an aberrant miss-firing of replication origins may explain non-recurrent rearrangements within the DMD.

Other regulatory regions have been characterized [13$18]$ as having a role in enhancing gene expression often in a tissue specific way.

The dystrophin coding regions share clear homology with at least three other classes of genes. The entire dystrophin coding sequence is similar to that of utrophin [19] while the $5^{\prime}$ end and central portions share homology with members of the spectrin gene family, including $\alpha$-actinin. The $3^{\prime}$ end of the dystrophin gene is homologous with an $87 \mathrm{kD}$ post-synaptic protein (dystrobrevin) characterized from the Torpedo electric organ. These observations suggest that dystrophin and utrophin may have arisen during evolution by juxtaposition of ancestral spectrin and $87 \mathrm{kD}$ like genes to form a larger transcription unit.

The cloning of the complete cDNA sequences encoding dystrophin [20] and utrophin [21] has given information on the predicted structure of these large cytoskeletal proteins. From their deduced amino-acid sequences both proteins share a surprisingly high number of identical residues $(46.3 \%)$. Dystrophin is a $427 \mathrm{kD}$ protein predicted to fold into several distinct structural domains. The amino terminus contains a major actin binding domain encoded by exons 1-8; however two further actin interactive sites have been mapped further $3^{\prime}$. The majority of the molecule is encoded by exons 9-63 forming the large rod-like domain composed of 24 "spectrin like" repeats interrupted by a few spacer regions. Located downstream of these "spectrin like" repeats is the cystein-rich domain (exons 64-69) which is followed by the C-terminus (exons 70-79), that binds to a group of transmembrane proteins as a complex originally named the dystrophin-associated-glycoprotein complex (DAG) because of its link to dystrophin. These proteins have been re-named dystrophin associated 
proteins (DAPs), also including a group of proteins located in the subsarcolemmal regions, such as sarcoglycans, $\alpha$-syntrophin and dystrobrevin [22].

The amino-terminus of dystrophin displays homology with a family of actin-binding proteins including $\alpha$-actinin and $\beta$-spectrin and this region is the one that interacts with the cytoskeleton. Three high-affinity actin-binding sites have been identified in the amino-terminus of dystrophin. However, surprisingly their deletion does not cause a major reduction of in vitro actin-binding affinity [23]. Confirming this view, some BMD patients have been reported as carrying deletions in this region $[24,25]$. These observations suggest that dystrophin may contain other actin-binding domains or alternatively it is able to associate with additional cytoskeletal proteins.

The central rod domain of dystrophin consists of 24 units homologous to repeat regions in the $\alpha$-spectrin and predicted to form triple helical coiled coils $[20,26]$. Each repeat is encoded by two exons, and one exon border is almost invariably located between repeat residues 47 and 48 of the 109 amino acid consensus sequences [26]. The repeats are interrupted by two non-helical regions that together with small regions separating these homologous units from neighboring domains are known as "hinges". Hinge 4 contains a WW domain whose function is still unknown but which is present in a variety of proteins from yeasts, nematodes and vertebrates [27]. The central hinges is thought to confer flexibility on the rod domain in response to muscle contraction [26].

At least a portion of the rod domain is present in all the dystrophin isoforms except for Dp71 which splits the WW motif in half. The WW domain locates in proximity to the dystrophin associated proteins (DAP) binding sites suggesting a possible role in contributing to protein-protein interactions.

Exons 64-69 encode the cystein-rich region. This amino acid region seems to stabilize dystroglycan binding [28].

Exons 71-74 encode the region shown to bind $\alpha-$ and $\beta$ syntrophin in vitro [29-31]. The fact that these exons are alternatively spliced suggests a potential role for this domain in modulating the functional interaction between dystrophin and syntrophin. The tissue specificity of the alternative splicing of these exons in brain [32] also reinforces this hypothesis. This dystrophin domain links to nitric oxide synthase (nNOS) via dystrobrevin. It is well known that in DMD, the absence of dystrophin at the sarcolemma delocalizes and down-regulates nNOS, altering HDAC2 chromatin association. An elegant paper demonstrated that HDAC2 nitrosylation state in DMD induce a deregulation of microRNA genes [33]. This suggests that the pathway activated by dystrophin/nNOS signaling controls muscle differentiation program.

The $3^{\prime}$ end of the alternative spliced region encodes the first two leucine zipper motifs, known to be involved in protein-protein interactions. There are 10 leucine zipper regions, four in the first motif and six in the second one. Four of the leucines in the second motif are capable of binding the troponin $\mathrm{T}$ in yeast [34].

The C-terminus of dystrophin is a 420 amino acid domain that has homology only to the utrophin gene.

Immunofluorescence microscopy has established dystrophin distribution at the plasma membrane of muscle

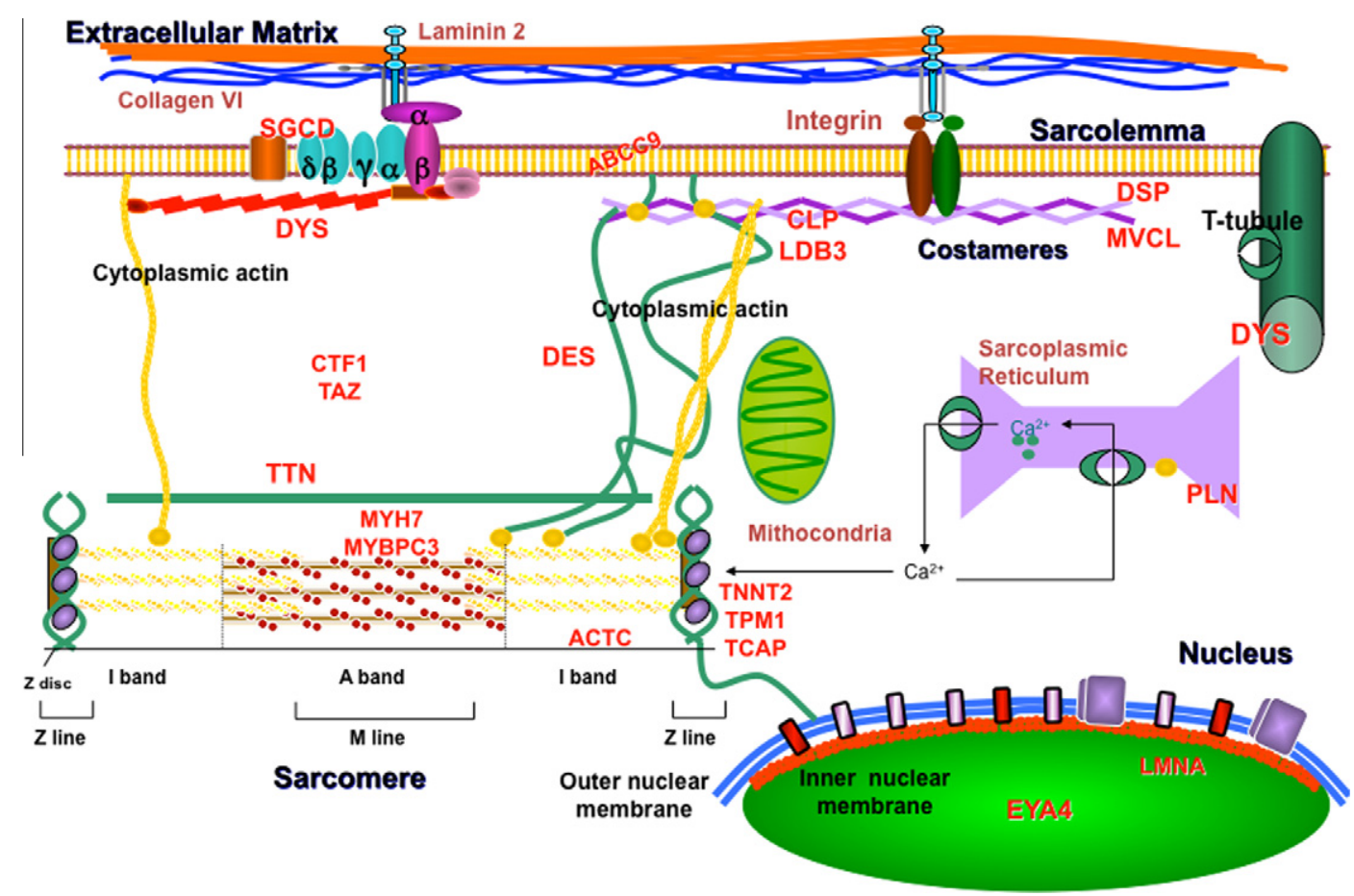

Fig. 2. Proteins and sarcolemma architecture. The structure of the sarcolemma and proteins participating to this specialized muscle membrane are shown. Intra- and extra-cellular compartment are also shown with the main proteins known to be related to a variety of muscle diseases (muscle dystrophies and myopathies). 
(cardiac, skeletal and smooth) and all muscle fibre types [30]. Biochemical preparations of sarcolemma confirmed that dystrophin copurifed with microsomes. Dystrophin is undetectable in the T-tubule system in skeletal muscle, although it is present in this system in the heart [35].

Freeze-etched and shadowed preparations of the inner surface of sarcolemma showed an interconnecting network of cytoskeletal proteins and these findings provided the first evidence that dystrophin has a spectrin-like function acting as a bridge anchoring protein and interconnecting the cytoskeleton to the extracellular matrix [30].

On the basis of dystrophin similarities to spectrin, Koenig et al., [20] predicted that it would form a flexible rod-like protein with a length of $125 \mathrm{~nm}$ which also forms side-by-side dimers and end-to end dimers or multimers. However, subsequent findings demonstrated that dystrophin is a monomer [36].

The full characterisation of dystrophin has provided more insight into the architecture of the sarcolemma and allowed us to define the cytoskeleton of muscle fibres. This is organized in functional distinct compartments and the sarcolemmal cytoskeleton itself can be broadly divided into junctional (myotendinous junction, neuromuscular junction and costamers or repetitive cytoskeletal units) and non-junctional (the muscular fiber and the costamers) domains [37]. Indeed, costamers share many similarities with junctional and non-junctional compartments because they are directly involved in the connection of the muscular fiber with the extracellular matrix. In junctional zones three different multimolecule complexes coexist: the focal adhesion-type, the spectrin-based and the dystrophin/ utrophin-based membrane skeleton systems. Their common features are the anchorage of actin filaments emanating from the intracytoplasmic level. The subsarcolemmal cytoskeleton complexes are thought to play distinct physiological roles in membrane stabilization, force transmission, ionic channel anchorage and perhaps other interrelated common functions, still unknown.

Many genes encoding specific proteins have been identified in this architecture and interestingly the majority of them can be mutated and then involved in different types of muscular dystrophy, supporting the cooperative nature of their functions in maintaining the sarcolemma integrity [38].

Fig. 2 is a representation of the sarcolemma organization with protein networks, and the known and hypothesized binding sites among these proteins.

\section{Mutations in the dystrophin gene and genotype/phenotype correlation}

The most common mutational event is represented by intragenic deletions accounting for the $65 \%$ of the dystrophin mutations. Duplications, accounts for the $10 \%$ of all mutations. Both rearrangements occur almost anywhere in the dystrophin gene; however two hot-spots (exon 3-7 and exons 44-53) are known. The genomic breakpoints of the $3^{\prime}$ hot spot frequently lying within intron 44 , while the $5^{\prime}$ end hot spot interests predominantly introns 2 and 7, those highly evolutionary conserved, and thought to contain regulatory regions. The other $25 \%$ circa of dystrophin mutations are represented by small mutations, including point mutations (missense, nonsense), frameshifting, indels, and other rare types (small inversion, complex small rearrangements). Atypical mutations (deep intronic, those rarely occurring in the $5^{\prime}$ or $3^{\prime}$ UTR regions) account for no more than $1 \%$.

The functional consequences of all mutation types are mainly related to the maintaining of the open reading frame, allowing a correct dystrophin to be translated [10]. So no relationship is present between the size, region, domain, mutations affecting splicing and the resultant clinical disease. Monaco et al., (1988) proposed that invariably DMD have out-of-frame mutations causing directly (nonsense) or indirectly (all types) premature stop codon downstream which determines too early truncated, nonfunctional protein. Oppositely, BMD is associated with in-frame mutations allowing the synthesis of a shorter but functionally preserved dystrophin.

This famous rule represents the rationale of the ongoing exon skipping approach, aiming at restoring dystrophin synthesis by re-establishing the frame (the so called BMD phenotype-reverting therapy, see below).

Functionally, the central and distal rod domains appear to be almost dispensable since deletions in this region are associated with high $\mathrm{CK}$, myalgia and muscle cramps but lack of weakness or only very mild signs [39-41].

This has been also demonstrated for patients with inframe deletions involving either exons $32-44$ or $48-51$ or $48-53$ all of which had normal or near normal dystrophin levels in the skeletal muscle [42].

In general, mutations which maintain the reading frame (in-frame) result in an abnormal, shorter but partially functional dystrophin and are associated with BMD. In DMD deletions and duplications disrupt the reading frame (frame-shift), resulting in unstable RNA that eventually leads to the production of virtually undetectable levels of truncated proteins. This phenomenon is called nonsense mediated decay (NMD), a process which deplete the major part of the dystrophin mRNA that contains out-of-frame mutations. The NMD is a vital mechanism able to eliminate the pathological allele mainly to avoid dominant negative effects. The mRNA that escapes this process may result in misfolded non-functional proteins that may also be mis-localized, unstable, determining heavy dominant negative effects [43].The reading frame hypothesis holds for over $90 \%$ of cases and is commonly used both as a diagnostic confirmation of dystrophinopathies but also for the differential diagnosis of DMD versus BMD.

Exceptions to the reading frame hypothesis do, however, exist and these involve both patients with BMD who carry frame-shift deletions/duplications or DMD with in-frame deletions/duplications.

An allelic phenotype of dystrophinopathies a part from BMD and DMD is the X-linked dilated cardiomyopathy 
(X-Linked Dilated Cardiomyopathy, XLDC, OMIM 302045).

This disease is characterized by selective cardiac involvement with no significant skeletal muscle symptoms [1]. Nonetheless, elevated serum creatine kinase (CK) values in this condition do suggest subclinical skeletal muscle involvement.

A classification of XLDC cases based on both molecular and clinical features has been proposed, distinguishing milder forms with delayed presentation associated with mutations in the spectrin-like dystrophin region ( $3^{\prime}$-XLDC) from the more severe early-onset forms caused by mutations in the $5^{\prime}$ region of dystrophin gene (5'-XLDC). Notably, a significant group of $5^{\prime}$-XLDC mutations specifically affects the expression of the M isoform [44]. Interestingly, all these different mutations share a common transcription pattern, characterized by an upregulation of the B (predominantly) and the $\mathrm{P}$ isoforms in the skeletal muscle of patients but not in the heart.

\section{Exceptions to the reading-frame hypothesis: BMD patients with frameshift mutations}

BMD patients with frame-shift mutations show a number of well-characterized deletions or duplications affecting the $5^{\prime}$ end of the gene (exons $3-7 ; 5-7 ; 3-6$ ) or further downstream (exons 51, 49-50, 47-52, 44 or 45 ). The most common event that allows these patients to produce at least some dystrophin is the exon skipping which occurs via alternative splicing; the carboxy-terminus is always preserved in these patients [45]. Diagnosis in such cases using a pure molecular genetic approach may be difficult, especially in young children without a positive family history, in that only a proportion with any one of these mutations will develop either BMD or DMD. The reason for the discrepancy of the observed phenotype is not fully understood and might be related to the efficiency and type of exon skipping events, which create larger messenger RNA deletions some of which are in-frame and therefore functional $[46,47]$. In these cases a muscle biopsy can help to verify if any dystrophin is produced or not and this information can be used together with the clinical assessment to predict the disease severity. An alternative mechanism for explaining the exception to the rule in BMD patients with outof-frame deletion involving exons 3-7 is the presence of an additional translation start site located within exon 8 . This hypothesis comes from RNA studies which failed to identify any exon skipping events in these BMD patients and showed the presence of a correct junction of exon 2 to exon 8 , predicting an out-of-frame messenger [48].

\section{Exon skipping events}

The mechanisms that lead to exon skipping in patients with out-of-frame mutations is still poorly understood and likely to be due to several factors. Exon skipping events limited to rare fibres are routinely found in the dystrophin deficient $m d x$ mouse (see below) and in approximately $50 \%$ of children with DMD $[46,49,50]$. In such cases the finding of revertant fibres in a patient is entirely compatible with a diagnosis of DMD. However several patients with out-of-frame mutations will be able to produce an appreciable amount of dystrophin either as a relatively low grade event in most fibres, or as an efficient mechanism in a proportion of fibres. While some exon skipping events occur more frequently in particular regions of the dystrophin gene, the type of exonic deletion does not necessarily predict if an exon skipping event will occur.

The induction of exon skipping using small molecules that affect dystrophin gene splicing is currently being carried out by several investigators in the hope that it will provide a possible therapeutic strategy in the future for patients with DMD.

\section{Exceptions to the reading-frame hypothesis: DMD patients with in-frame deletion mutations}

While there is often a correlation between disease phenotype and the ability to produce dystrophin it is important to be aware that there are rare exceptions. This is particularly true for large deletions in the $5^{\prime}$ region that extend into the mid rod domain, for example deletions removing exons $3-31,3-25,4-41$, or 4-18 [51]. This contrasts with the mild BMD phenotype observed for large deletions that do not involve the $5^{\prime}$ principal putative actin binding site of dystrophin but instead remove regions of the rod domain [52-55]. As a general rule it seems that deletions of large portions of the rod domain can result in a $\mathrm{BMD}$, as long as the $\mathrm{N}$-and $\mathrm{C}$-terminus of dystrophin are maintained [45]. The functional relevance of a preserved actin binding domain is also highlighted by relatively small in-frame deletions that however disrupt the $5^{\prime}$ actin binding domain, such as the deletion of exons 3-13: these are usually associated with a DMD phenotype [56]. An useful and extensive table with the phenotypes observed in patients with a variety of deletions can be found in the Leiden Duchenne Muscular Dystrophy database (http://www. dmd.nl/).

A more complete understanding of the functional domains of the dystrophin protein is not only important for the diagnosis of dystrophinopathies but is being exploited by investigators involved in the development of gene therapy approaches for DMD [57-59].

\section{Point mutations}

Up to $20-35 \%$ of patients with DMD and BMD do not have deletions or duplications of the dystrophin gene [60]. In a routine diagnostic context, the identification of mutations in these patients can be complicated/slowed by the huge size of the dystrophin gene and so specialized techniques have been used [61]. Most of the mutations identified in DMD are nonsense point mutations or small 


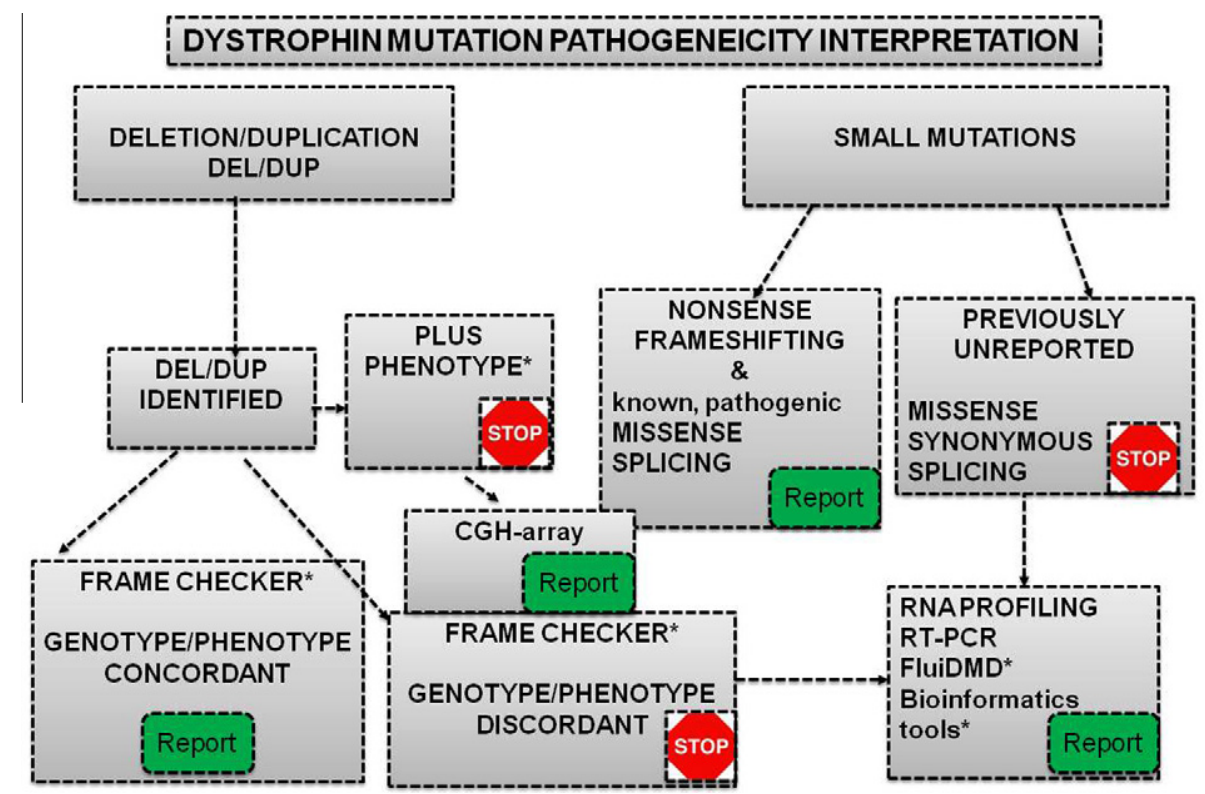

Fig. 3. Dystrophin mutations' interpretation. A flowchart of the procedures and tools for the correct and accurate mutation interpretation meaning is shown. Abbreviation: MLPA, multiple ligation probe assay; CGH, comparative genomic hybridization; the symbol STOP means that further actions are required in order to complete the genetic diagnosis, the symbol REPORT means that the diagnostic report can be delivered (the pathogenic meaning of the mutation is certain) (See Appendix A for further details).

frameshifting deletions/insertions, followed by splice site mutations. It should be stressed on the other hand that some missense or nonsense mutations, are indeed capable of inducing exon skipping. Indeed, missense mutations may occur within exonic splicing enhancers (ESEs), which represent motifs participating to the process of exon definition. Therefore missense mutations can disrupt ESEs and then can affect the splicing profile of the dystrophin gene $[62,63]$. This is why the study of the RNA/protein profile in skeletal muscle cells is of high relevance for accurate prognostic prediction and improved understanding of disease pathogenesis in individual cases.

Guidelines for dystrophinopathies molecular diagnosis have been recently published [64] but over the next few years next generations sequencing and related methodology will transform the genetic definition of genetic disease, including dystrophinopathies.

Fig. 3 shows a proposed flowchart of the procedures and tools for the correct and accurate mutation interpretation.

\section{Interpreting functional meaning of dystrophin mutations}

The great mutation heterogeneity largely complicates the genetic definition in dystrophinopathies, and multiple approaches/techniques, applied according to an established flow-chart based on mutation frequencies and clinical data, are required to reach an high diagnostic sensitivity [64].

Currently, the combined use of all available techniques for DMD gene mutation screening allows to reach a genetic definition in almost all cases. Nevertheless, problems can arise when the functional meaning of DMD identified mutations has to be decoded. Apparently easily interpretable mutations often show not obvious and unpredictable effects on phenotype.

This is the case, for example, of nonsense mutations occurring within DMD muscle isoform exons 1 that have been associated to a very mild BMD phenotype with deambulation preserved into the seventh decade.

It has been proposed, based on in vitro studies, these mutations being ameliorated by internal initiation at two AUG codons identified within exon 6 [11]. Similarly to BMD-causing exon 3-7 deletion, representing the most classical example of out-of-frame mutation escaping Monaco's rule, the nonsense mutations within DMD exon 1 highlight the relevance of a comprehensive medical genetic approach to DMD mutation interpretation, strongly integrating molecular and clinical data.

Other peculiar DMD mutations require higher levels of laboratory investigation to be clarified in their functional meaning. An out-of-frame duplication of exons 3-6, unexpectedly associating with a mild phenotype, was shown by RNA studies to be entirely transcribed in an in-frame exons 3-5 duplicated transcript, thus explaining the BMD phenotype [65]. Array-CGH analysis enabled us to determine that the duplication breakpoint was within the coding sequence of exon 6, thus justifying the noninclusion of a copy of exon 6 into the dystrophin transcript [66].

In other cases, the reasons for the discrepancies between predicted mutation effect and observed phenotype remain substantially unexplained. RNA studies performed in an exon 14-22 in-frame duplication observed in a DMD patient and in an out-of-frame exon 2 and exons 51-55 duplications occurring in BMD patients, failed to 
demonstrate splicing variants [65-67]. In all these cases the transcriptional profile was consistent with the genomic mutations, evoking the involvement of undefined posttranscriptional mechanisms affecting protein function, as cause of the discrepant phenotype.

DMD mutations that primarily warrants demonstration of a functional meaning before being released as pathogenic are deep intronic rearrangements, currently easily identified by the development of high density microarraybased $\mathrm{CGH}$ approach exploring the full genomic region of the gene $[66,68,69]$. RNA analysis is mandatory in these cases: a $1.3 \mathrm{~kb}$ duplication deeply embedded within intron 4 was shown to cause retention of intronic sequences in the mature DMD transcript and classified as pathogenic [66]; an inversion within intron 44 flanked by two $52 \mathrm{~Kb}$ and $1 \mathrm{~Kb}$ deleted segments identified by array-CGH was shown to cause a pathological pseudo-exon inclusion within dystrophin RNA [70].

Mutations in introns that cause misplicing by inducing inclusion of intronic sequence as exons (pseudo-exon inclusion) represent a peculiar class of disease causing mutations not rare among dystrophinopathies. About 30 cases of pathological pseudoexon inclusion in the DMD gene are reported in locus specific databases (http://www.umd. be.DMD/ and http://www.dmd.nl). Apart from the few cases related to genomic rearrangements, most of them result from single nucleotide substitution strengthening pre-existing splice sites or creating new cryptic ones [66,7173]. These mutations escape DNA-based techniques exploring coding regions, further highlighting RNA analysis as a crucial step for a comprehensive diagnostic strategy in dystrophinopathies.

An innovative tool able to explore the full dystrophin messenger consists of the FluiDMD [74]. This has revealed valuable both for mutation identification at the RNA level and for profiling the mutation impact at the RNA level.

\section{Double troubles in dystrophinopathies}

A peculiar aspect in dystrophinopathies is the occurrence of more than one DMD mutation within the same pedigree, observed in up to $3 \%$ of familial DMD cases [75-78]. These atypical genealogies may present as DMD cases related trough paternal line or as affected boys related trough maternal line but carrying different mutations. In both cases independent mutational events are implied, suggesting the existence of peculiar genomic configurations within the DMD locus predisposing to an increased mutation rate in some families. A transposon-mediated mechanism has been hypothesised, also supported by different reports of LINE-1 related DMD mutations [79,80]. We also have observed a family with recurrence of a BMD phenotype in two males maternally related but carrying two different mutations, an exon 61-63 deletion and an exon 45-48 deletion. The exon 61-63 deletion has arisen de novo in the mother of the affected male, whereas the origin of the 45-48 deletion is unknown. Fig. 4 shows the pedigree with recurrence of a BMD phenotype in this family due to two different mutations.

We also observed a family with XLDC recurrence in two male cousins which however were again carrying two different mutations, a point mutation at the donor splice site of muscle intron 1 and a deletion of exons 49-51. Genetic analysis in this pedigree revealed that the two mutations were originated from different, unrelated ancestors. Even if relatively uncommon, the possibility of different mutations occurring in the same family suggests the opportunity to genotype all affected individuals in a pedigree and have to be considered when counseling individuals at risk for a known familial DMD mutation. In these cases, at least for deletions/duplications, requiring rapid and easy procedure to be investigated (as MLPA), the full screening of the $79 \mathrm{DMD}$ exons is probably preferable to the testing of specific familial mutations.

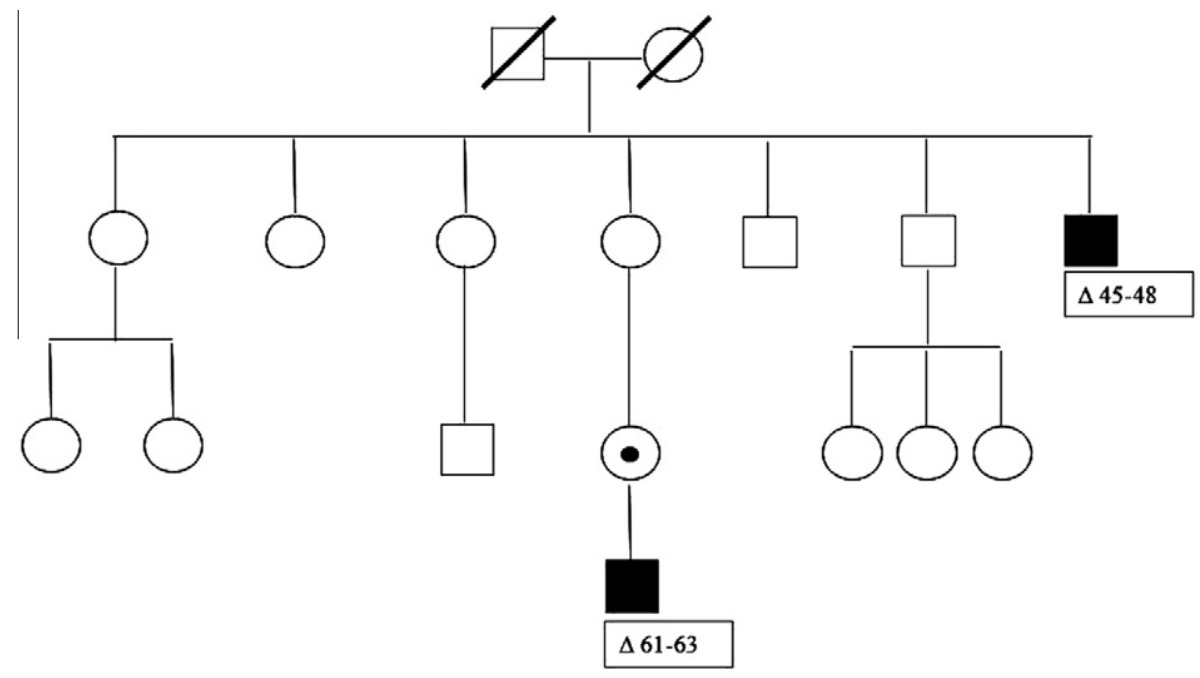

Fig. 4. A double trouble pedigree. Pedigree with recurrence of a BMD phenotype in two males maternally related but carrying two different mutations, an exon 61-63 deletion and an exon 45-48 deletion. 
This is even more true when considering that two different mutations in a single dystrophin gene have been also reported as "double trouble" in dystrophinopathies. A BMD patient has been described carrying a point mutation affecting exon 19 splicing and a deletion of exons 46-48 [81]. A double-duplication in two DMD-affected brothers and in their carrier mother was also reported which consist of two non-contiguous duplications encompassing exons 2-7 and exons 50-55 [82].

We also have identified a female carrying two noncontiguous duplications within the dystrophin gene, one involving Purkinje exon 1-exon 7 and the second including exons 13-42. The female was asymptomatic, indicating that the two duplications were in cis, thus on the same mutated $\mathrm{X}$-chromosome [83]. Recently a novel complex rearrangement was described in a DMD patient consisting of a $90 \mathrm{~kb}$ insertion of non-contiguous sequences belonging to chromosome 4 within DMD intron 43 [84]. This rearrangement escaped routine DNA-based diagnostic procedures and was identified by RNA studies, revealing the insertion of a cryptic exon between DMD exons 43 and 44 originating from chromosome 4 . This family, enlarging the spectrum of mutational mechanisms in the DMD gene, further emphasize the importance of a comprehensive molecular diagnosis in these patients.

\section{Clinical and Therapeutic Biomarkers in dystrophinopathies}

The dystrophinopathies can be considered a group of different phenotypes all due to dystrophin mutations. The biological reasons for this phenotypic variability are still unknown. In addition, clinical variability was observed among the DMD patients in terms of ambulation loss, age length (supersurvivors), cardiomyopathy, and also drug response (steroids) [85]. It is therefore very likely that other modifiers (either genetics and environmental) may contribute to these differences. Biomarkers are measurable biological parameters able to quantify and monitor disease progression, disease course and drug response and other crucial disease signs [86]. There is general agreement that finding biomarkers, especially low invasive, is mandatory for repeated checks and monitoring of DMD and, more in general, rare diseases.

Very recently, biomarkers discovery raises interest within DMD and the first exploratory genetic modifiers of muscular dystrophy in humans were identified $[87,88]$. A common DNA variant in the promoter sequence of the SSP1 gene, encoding osteopontin, was shown to affect both disease severity and response to corticosteroids in DMD boys. The G allele of SSP1 polymorphism, despite its magnitude of effect still to be established, was shown to be associated with more severe disease progression. This evidence, if confirmed in larger cohorts, will have great impact both on patients selection/stratification for clinical incoming trials and for uncovering novel therapeutic targets in dystrophinopathies. MMP-9 level in sera of DMD patients were higher in respect to controls, and also correlate significantly with disease severity in advanced stages. Further studies are required in order to proof that these biomarkers can be used to monitor disease severity. Bioinformatics approaches are also needed in order to speed up and facilitate the biomarker discovery phase. Kotelnikova et al., [89] have developed an algorithm able to identify candidate biomarkers by literature analysis. This interesting novel approach finds possible applications in all neuromuscular disorders.

\section{Mutations and personalized medicine: back to genetics}

In the last few years we experienced a flowering of novel therapeutic options for DMD beside the well recognized corticosteroid treatment, and among various appealing pre-clinical studies, exon skipping approach for reframing dystrophin, stop codon reversion using gentamycin and ataluren have been translated into clinical trials $[90,91]$. Promising results came from antisense therapy, using differently backboned antisense oligonucleotides (phosphorothioate and morpholino) and different administration routes (subcutaneous and intravenous) $[92,93]$. All these trials are mutation specific, meaning that the drug (the antisense molecule) can be used for skipping the exon (and only that) which further omission from the transcript reframes dystrophin. PTC124 (ataluren) reverts primary stop codon thus it can be used only in DMD patients with stop mutations (and not frameshifting, any type). Therefore fine genetic characterization has become mandatory for making patients eligible for novel personalized trials. This fact has greatly increased the requests of molecular genetic diagnosis, with a remarkable heavy overload of the molecular genetic laboratories around the world, especially for the heavy full exons sequencing. Since DMD and dystrophin gene diagnostics still remain a high demanding task and guidelines have been recently defined, the novel high throughput methods will surely speed up and improve the accuracy and sensitivity of molecular diagnosis [94].

\section{Conclusions}

Since the identification of the dystrophin gene, enormous progresses have been made through the years resulting in a detailed clinical definition of the disease, in molecular testing strategies and prevention, in the comprehension of the different genotype phenotype correlations and in novel trials. Recently, novel reports on neonatal screening for DMD have been published, raising scientific and ethical issues and needing the setting of national programs to be fully and successfully adopted $[95,96]$. We have learned that dystrophinopathies' molecular genetics is governed by rules but also shows exception to the rules and these latter have always to be taken into account and deserve extreme accuracy in the diagnostic strategy, also in view of the novel personalized trials 
ongoing. A part from dystrophin, which plays the lead role, the discovery of biomarkers as modifiers of the disease is broadening the scenario and addressing the effort also through the identification of new interacting players; this will represent a powerful tool for a tailored medicine in order to monitor the progression of the disease, the response to the therapy and to adopt early efficacious interventions.

\section{Acknowledgments}

A.F, M.N, and F.G work is supported by the BIO-NMD project (EU 241665), by the Telethon Italy Foundation grants GGPO5115, GGPU7011, and GGPO9093, and by the Duchenne Parent Project Italy. $\mathrm{AF}$ is member of the Research Committee of the European NeuroMuscularCentre.

\section{Appendix A}

Specifications $(*)$ :

-bioinformatics tools*: some mutations require a bioinformatics analysis using online freely available tools as: Mutation taster: http://www.mutationtaster.org/ BDGP splice site prediction: http://www.fruitfly.org/ seq_tools/splice.html

ESE FINDER: http://rulai.cshl.edu/cgi-bin/tools/ ESE3/esefinder.cgi?process $=$ home

ASSA (Automated Splice Site Analysis): https:// splice.uwo.ca/

PolyPhen-2: http://genetics.bwh.harvard.edu/pph2/

SIFT: http://sift.jcvi.org/

These informatics tools can orient the mutation interpretation for further analysis, but can never be considered as diagnostics alone.

-Frame Checker*: at the Leiden DMD pages (http:// www.dmd.nl/) the frame checker function can be found. Using this on-line application, it is easy to establish if the deletion mutation is in-frame or out-of-frame. The frame rule and frame checker can be applied also to duplications, taking into account the exception to the rules; it can be hardly applied to very large duplications, that, though in-frame, are often associated to a DMD phenotype.

-Plus phenotype* : if the phenotype is atypical (mental retardation, other unusual signs or symptoms) it is appropriate to carry on a CGH array analysis (both whole genome and dystrophin locus specific) for ruling out complex rearrangements within dystrophin itself or even involving multiple loci.

-FluiDMD*: this is novel tool which is able to explore the full dystrophin RNA profile and exon composition and is also useful for mutation meaning confirmation at the RNA level [74].

\section{References}

[1] Muntoni F, Torelli S, Ferlini A. Dystrophin and mutations: one gene, several proteins, multiple phenotypes. Lancet Neurol 2003;2:731-40.

[2] Udd B. Third filament diseases. Adv Exp Med Biol 2008;642:99-115.

[3] Bies RD, Phelps SF, Cortez MD, Roberts R, Caskey CT, Chamberlain JS. Human and murine dystrophin mRNA transcripts are differentially expressed during skeletal muscle, heart, and brain development. Nucleic Acids Res 1992;20:1725-31.

[4] Torelli S, Ferlini A, Obici L, Sewry C, Muntoni F. Expression, regulation and localisation of dystrophin isoforms in human foetal skeletal and cardiac muscle. Neuromuscul Disord 1999;9(8):541-51.

[5] Mioni F, Danieli GA, Cao A, et al. A report on 528 intragenic deletions detected in DMD and BMD patients by an Italian collaborative study. Gene Geogr 1994;8(1):35-44.

[6] Nobile C, Galvagni F, Marchi J, Roberts R, Vitiello L. Genomic organization of the human dystrophin gene across the major deletion hot spot and the $3^{\prime}$ region. Genomics 1995;28(1):97-100.

[7] McNaughton JC, Hughes G, Jones WA, Stockwell PA, Klamut HJ, Petersen GB. The evolution of an intron: analysis of a long, deletionprone intron in the human dystrophin gene. Genomics 1997;40(2): 294-304.

[8] McNaughton JC, Cockburn DJ, Hughes G, et al. Is gene deletion in eukaryotes sequence-dependent? A study of nine deletion junctions and nineteen other deletion breakpoints in intron 7 of the human dystrophin gene. Gene 1998;222(1):41-51.

[9] Nachman MW, Crowell SL. Contrasting evolutionary histories of two introns of the Duchenne muscular dystrophy gene, Dmd, in humans. Genetics 2000;155(4):1855-64.

[10] Monaco AP, Bertelson CJ, Liechti-Gallati S, Moser H, Kunkel LM. An explanation for the phenotypic differences between patients bearing partial deletions of the DMD locus. Genomics 1988;2:90-5.

[11] Gurvich OL, Maiti B, Weiss RB, Aggarwal G, Howard MT, Flanigan KM. DMD exon 1 truncating point mutations: amelioration of phenotype by alternative translation initiation in exon 6. Hum Mutat 2009;30(4):633-40.

[12] Ankala A, Kohn JN, Hegde A, et al. Aberrant firing of replication origins potentially explains intragenic nonrecurrent rearrangements within genes, including the human DMD gene. Genome Res 2012;22(1):25-34.

[13] Ogawa M, Kaname T, Kimura S, et al. The lacZ gene under the control of the $7 \mathrm{~kb}$ of human dystrophin muscle specific promoter is expressed in cardiac muscle but not in adult skeletal muscle in transgenic mice. Neuromuscul Disord 2001;11:244-50.

[14] Shiga N, Takeshima Y, Sakamoto H, et al. Disruption of the splicing enhancer sequence within exon 27 of the dystrophin gene by a nonsense mutation induces partial skipping of the exon and is responsible for Becker muscular dystrophy. J Clin Invest 1997;100(9): 2204-10.

[15] Klamut HJ, Gangopadhyay SB, Worton RG, Ray PN. Molecular and functional analysis of the muscle-specific promoter region of the Duchenne muscular dystrophy gene. Mol Cell Biol 1990;10:193-205.

[16] Klamut HJ, Bosnoyan-Collins LO, Worton RG, Ray PN, Davis HL. Identification of a transcriptional enhancer within muscle intron 1 of the human dystrophin gene. Hum Mol Genet 1996;5(10):1599-606.

[17] Klamut HJ, Bosnoyan-Collins LO, Worton RG, Ray PN. A musclespecific enhancer within intron 1 of the human dystrophin gene is functionally dependent on single MEF-1/E box and MEF-2/AT-rich sequence motifs. Nucleic Acids Res 1997;5:1618-25.

[18] Fracasso C, Patarnello T. Evolution of the dystrophin muscular promoter and $5^{\prime}$ flanking region in primates. J Mol Evol 1998;46(2):168-79.

[19] Gualandi F, Rimessi P, Trabanelli C, et al. Intronic breakpoint definition and transcription analysis in DMD/BMD patients with deletion/duplication at the $5^{\prime}$ mutation hot spot of the dystrophin gene. Gene 2006;370:26-33. 
[20] Koenig M, Monaco AP, Kunkel LM. The complete sequence of dystrophin predicts a rod-shaped cytoskeletal protein. Cell 1988;53(2):219-28.

[21] Tinsley JM, Blake DJ, Roche A, et al. Primary structure of dystrophin-related protein. Nature 1992;360:591-3.

[22] Tinsley JM, Blake DJ, Pearce M, Knight AE, Kendrick-Jones J, Davies KE. Dystrophin and related proteins. Curr Opin Genet Dev 1993;3(3):484-90.

[23] Corrado K, Mills PL, Chamberlain JS. Deletion analysis of the dystrophin-actin binding domain. FEBS Lett 1994;344(2-3):255-60.

[24] Malhotra SB, Hart KA, Klamut HJ, et al.. Frame-shift deletions in patients with Duchenne and Becker muscular dystrophy. Science 1988;242(4879):755-9.

[25] Beggs AH, Hoffman EP, Snyder JR, et al. Exploring the molecular basis for variability among patients with Becker muscular dystrophy: dystrophin gene and protein studies. Am J Hum Genet 1991;49(1):54-67.

[26] Koenig M, Kunkel LM. Detailed analysis of the repeat domain of dystrophin reveals four potential hinge segments that may confer flexibility. J Biol Chem 1990;265(8):4560-6.

[27] André B, Springael JY. WWP, a new amino acid motif present in single ormultiple copies in various proteins including dystrophin and the SH3-bindingYes-associated protein YAP65. Biochem Biophys Res Commun 1994;205(2):1201-5.

[28] Jung D, Yang B, Meyer J, Chamberlain JS, Campbell KP. Identification and characterization of the dystrophin anchoring site on betadystroglycan. J Biol Chem 1995;270(45):27305-10.

[29] Yang B, Jung D, Rafael JA, Chamberlain JS, Campbell KP. Identification of alpha-syntrophin binding to syntrophin triplet, dystrophin, and utrophin. J Biol Chem 1995;270(10):4975-8.

[30] Ahn AH, Kunkel LM. Syntrophin binds to an alternatively spliced exon of dystrophin. J Cell Biol 1995;128(3):363-71.

[31] Kramarcy NR, Vidal A, Froehner SC, Sealock R. Association of utrophin and multiple dystrophin short forms with the mammalian $\mathrm{M}(\mathrm{r})$ 58,000 dystrophin-associated protein (syntrophin). J Biol Chem 1994;269(4):2870-6.

[32] Feener CA, Koenig M, Kunkel LM. Alternative splicing of human dystrophin mRNA generates isoforms at the carboxy terminus. Nature 1989;338:509-11.

[33] Cacchiarelli D, Martone J, Girardi E, et al. MicroRNAs involved in molecular circuitries relevant for the Duchenne muscular dystrophy pathogenesis are controlled by the dystrophin/nNOS pathway. Cell Metab 2010;12(4):341-51.

[34] Pearlman JA, Powaser PA, Elledge SJ, Caskey CT. Troponin T is capable of binding dystrophin via a leucine zipper. FEBS Lett 1994;354(2):183-6.

[35] Kaprielian RR, Stevenson S, Rothery SM, Cullen MJ, Severs NJ. Distinct patterns of dystrophin organization in myocyte sarcolemma and transverse tubules of normal and diseased human myocardium. Circulation 2000;101(22):2586-94.

[36] Ahn AH, Kunkel LM. The structural and functional diversity of dystrophin. Nat Genet 1993;3:283-91.

[37] Berthier C, Blaineau S. Supramolecular organization of the subsarcolemmal cytoskeleton of adult skeletal muscle fibers. A Rev Biol Cell 1997;89(7):413-34, Review.

[38] Nigro V, Aurino S, Piluso G. Limb girdle muscular dystrophies: update on genetic diagnosis and therapeutic approaches. Curr Opin Neurol 2011;24(5):429-36, Review.

[39] Angelini C, Fanin M, Freda MP, et al. Prognostic factors in mild dystrophinopathies. J Neurol Sci 1996;142(1-2):70-8.

[40] Beggs AH, Hoffman EP, Snyder JR, et al. Exploring the molecular basis for variability among patients with Becker muscular dystrophy: dystrophin gene and protein studies. Am $\mathrm{J}$ Hum Genet 1991;49(1):54-67.

[41] Gospe Jr SM, Lazaro RP, Lava NS, Grootscholten PM, Scott MO, Fischbeck KH. Familial X-linked myalgia and cramps: a nonprogressive myopathy associated with a deletion in the dystrophin gene. Neurology 1989;39(10):1277-80.
[42] Melis MA, Cau M, Muntoni F, et al. Elevation of serum creatine kinase as the only manifestation of an intragenic deletion of the dystrophin gene in three unrelated families. Eur J Paediatr Neurol 1998;2:255-61.

[43] Mühlemann O, Eberle AB, Stalder L, Zamudio Orozco R. Recognition and elimination of nonsense mRNA. Biochim Biophys Acta 2008;1779(9):538-49.

[44] Neri M, Valli E, Alfano G, et al. The absence of dystrophin brain isoform expression in healthy human heart ventricles explains the pathogenesis of $5^{\prime} \mathrm{X}$-linked dilated cardiomyopathy. BMC Med Genet 2012;28(13):20.

[45] Arahata K, Beggs AH, Honda H, et al. Preservation of the Cterminus of dystrophin molecule in the skeletal muscle from Becker muscular dystrophy. J Neurol Sci 1991;101:148-56.

[46] Nicholson LV, Bushby KM, Johnson MA, den Dunnen JT, Ginjaar IB, van Ommen GJ. Predicted and observed sizes of dystrophin in some patients with gene deletions that disrupt the open reading frame. J Med Genet 1992;29:892-6.

[47] Patria SY, Alimsardjono H, Nishio H, Takeshima Y, Nakamura H, Matsuo M. A case of Becker muscular dystrophy resulting from the skipping of four contiguous exons (71-74) of the dystrophin gene during mRNA maturation. Proc Assoc Am Phys 1996;108:308-14.

[48] Winnard AV, Mendell JR, Prior TW, Florence J, Burghes AH. Frameshift deletions of exons 3-7 and revertant fibers in Duchenne muscular dystrophy: mechanisms of dystrophin production. Am J Hum Genet 1995;56:158-66.

[49] Sherratt TG, Vulliamy T, Dubowitz V, Sewry CA, Strong PN. Exon skipping and translation in patients with frameshift deletions in the dystrophin gene. Am J Hum Genet 1993;53:1007-15.

[50] Prior TW, Bartolo C, Papp AC, et al. Dystrophin expression in a Duchenne muscular dystrophy patient with a frame shift deletion. Neurology 1997;48:486-8.

[51] Nevo Y, Muntoni F, Sewry C, et al. Large in-frame deletions of the rod-shaped domain of the dystrophin gene resulting in severe phenotype. Isr Med Assoc J 2003;5:94-7.

[52] Love DR, Flint TJ, Marsden RF, et al. Characterization of deletions in the dystrophin gene giving mild phenotypes. Am J Med Genet 1990;37:136-42.

[53] Bushby KM, Gardner-Medwin D, Nicholson LV, et al. The clinical, genetic and dystrophin characteristics of Becker muscular dystrophy, II: correlation of phenotype with genetic and protein abnormalities. J Neurol 1993;240:105-12.

[54] England SB, Nicholson LV, Johnson MA, et al. Very mild muscular dystrophy associated with the deletion of $46 \%$ of dystrophin. Nature 1990;343:180-2.

[55] Love DR, Flint TJ, Genet SA, Middleton-Price HR, Davies KE. Becker muscular dystrophy patient with a large intragenic dystrophin deletion: implications for functional minigenes and gene therapy. J Med Genet 1991;28:860-4.

[56] Muntoni F, Gobbi P, Sewry C, et al. Deletions in the $5^{\prime}$ region of dystrophin and resulting phenotypes. J Med Genet 1994;31:843-7.

[57] Roberts M, Dickson G. The future of Duchenne muscular dystrophy gene therapy: shrinking the dystrophin gene. Curr Opin Mol Ther 2002;4:343-8

[58] Wells DJ, Wells KE, Asante EA, et al. Expression of human fulllength and minidystrophin in transgenic mdx mice: implications for gene therapy of Duchenne muscular dystrophy. Hum Mol Genet 1995;4:1245-50.

[59] Harper SQ, Hauser MA, DelloRusso C, et al. Modular flexibility of dystrophin: implications for gene therapy of Duchenne muscular dystrophy. Nat Med 2002;8:253-61.

[60] Roberts RG, Gardner RJ, Bobrow M. Searching for the 1 in 2,400,000: a review of dystrophin gene point mutations. Hum Mutat 1994;4:1-11.

[61] Flanigan KM, Dunn DM, von Niederhausern A, et al. Mutational spectrum of DMD mutations in dystrophinopathy patients: application of modern diagnostic techniques to a large cohort. Hum Mutat 2009;30(12):1657-66. 
[62] Ginjaar IB, Kneppers AL, V d Meulen JD, et al. Dystrophin nonsense mutation induces different levels of exon 29 skipping and leads to variable phenotypes within one BMD family. Eur J Hum Genet 2000;8:793-6.

[63] Shiga N, Takeshima Y, Sakamoto H, et al. Disruption of the splicing enhancer sequence within exon 27 of the dystrophin gene by a nonsense mutation induces partial skipping of the exon and is responsible for Becker muscular dystrophy. J Clin Invest 1997;100:2204-10.

[64] Abbs S, Tuffery-Giraud S, Bakker E, Ferlini A, Sejersen T, Mueller CR. Best practice guidelines on molecular diagnostics in Duchenne/ Becker muscular dystrophies. Neuromuscul Disord 2010;20(6):422-7.

[65] Gualandi F, Neri M, Bovolenta M, et al.. Transcriptional behavior of DMD gene duplications in DMD/BMD males. Hum Mutat 2009;30(2):E310-9.

[66] Bovolenta M, Neri M, Fini S, et al. A novel custom high densitycomparative genomic hybridization array detects common rearrangements as well as deep intronic mutations in dystrophinopathies. BMC Genomics 2008;28(9):572.

[67] Kesari A, Pirra LN, Bremadesam L, et al. cDNA, and protein studies in Becker muscular dystrophy show high exception to the reading frame rule. Hum Mutat 2008;29(5):728-37.

[68] del Gaudio D, Yang Y, Boggs BA, et al. Molecular diagnosis of Duchenne/Becker muscular dystrophy: enhanced detection of dystrophin gene rearrangements by oligonucleotide array-comparative genomic hybridization. Hum Mutat 2008;29(9):1100-7.

[69] Hegde MR, Chin EL, Mulle JG, Okou DT, Warren ST, Zwick ME. Microarray-based mutation detection in the dystrophin gene. Hum Mutat 2008;29(9):1091.

[70] Khelifi MM, Ishmukhametova A, Khau Van Kien P, et al. Pure intronic rearrangements leading to aberrant pseudoexon inclusion in dystrophinopathy: a new class of mutations? Hum Mutat 2011;32(4):467-75.

[71] Gurvich OL, Tuohy TM, Howard MT, et al. DMD pseudoexon mutations: splicing efficiency, phenotype, and potential therapy. Ann Neurol 2008;63(1):81-9.

[72] Takeshima Y, Yagi M, Okizuka Y, et al. Mutation spectrum of the dystrophin gene in 442 Duchenne/Becker muscular dystrophy cases from one Japanese referral center. J Hum Genet 2010;55(6):379-88.

[73] Dhir A, Buratti E. Alternative splicing: role of pseudoexons in human disease and potential therapeutic strategies. FEBS J 2010;277(4):841-55.

[74] Bovolenta M, Scotton C, Falzarano MS, Gualandi F, Ferlini A. Rapid, comprehensive analysis of the dystrophin transcript by a custom micro-fluidic exome array. Hum Mutat 2012;33(3):572-81.

[75] Miciak A, Keen A, Jadayel D, Bundey S. Multiple mutations in an extended Duchenne muscular dystrophy family. J Med Genet 1992;29:123-6.

[76] Mostacciuolo ML, Miorin M, Vitiello L, et al. Occurrence of two different intragenic deletions in two male relatives affected with Duchenne muscular dystrophy. Am J Med Genet 1994;50(1):84-6.

[77] Morandi L, Mora M, Tedeschi S, et al.. DMD and BMD in the same family due to distinct mutations. Am J Med Genet 1995;59(4):501-5.

[78] Zatz M, Passos-Bueno MR, Rapaport D, Vainzof M. Familial occurrence of Duchenne dystrophy through paternal lines in four families. Am J Med Genet 1991;38(1):80-4.

[79] Narita N, Nishio H, Kitoh Y, et al. Insertion of a $5^{\prime}$ truncated L1 element into the $3^{\prime}$ end of exon 44 of the dystrophin gene resulted in skipping of the exon during splicing in a case of Duchenne muscular dystrophy. J Clin Invest 1993;91(5):1862-7.

[80] Pizzuti A, Pieretti M, Fenwick RG, Gibbs RA, Caskey CT. A transposon-like element in the deletion-prone region of the dystrophin gene. Genomics 1992;13(3):594-600.

[81] Wilton SD, Johnsen RD, Pedretti JR, Laing NG. Two distinct mutations in a single dystrophin gene: identification of an altered splice-site as the primary Becker muscular dystrophy mutation. Am J Med Genet 1993;46(5):563-9.

[82] Fenollar-Cortés M, Gallego-Merlo J, Trujillo-Tiebas MJ, LordaSánchez I, Ayuso C. Two non-contiguous duplications in the DMD gene in a Spanish family. J Neurogenet 2008;22(1):93-101.

[83] Brioschi S, Gualandi F, Scotton C, et al. Genetic characterization in symptomatic female DMD carriers: lack of relationship between Xinactivation, transcriptional DMD allele balancing and phenotype. BMC Med Genet 2012;13(1):73.

[84] Baskin B, Gibson WT, Ray PN. Duchenne muscular dystrophy caused by a complex rearrangement between intron 43 of the DMD gene and chromosome 4. Neuromuscul Disord 2011;21(3): 178-82.

[85] Desguerre I, Christov C, Mayer M, et al. Clinical heterogeneity of Duchenne muscular dystrophy (DMD): definition of sub-phenotypes and predictive criteria by long-term follow-up. PLoS One 2009;4(2):e4347.

[86] Gurwitz D, Lunshof J. Personalized pharmacotherapy: genotypes, biomarkers, and beyond. Clin Pharmacol Ther 2008.

[87] Nadarajah VD, van Putten M, Chaouch A, et al. Serum matrix metalloproteinase-9 (MMP-9) as a biomarker for monitoring disease progression in Duchenne muscular dystrophy (DMD). Neuromuscul Disord 2011;21(8):569-78.

[88] Pegoraro E, Hoffman EP, Piva L, et al. SPP1 genotype is a determinant of disease severity in Duchenne muscular dystrophy. Neurology 2011;76(3):219-26.

[89] Kotelnikova E, Shkrob MA, Pyatnitskiy MA, Ferlini A, Daraselia N. Novel approach to meta-analysis of microarray datasets reveals muscle remodeling-related drug targets and biomarkers in Duchenne muscular dystrophy. PLoS Comput Biol 2012;8(2).

[90] Muntoni F, Wood MJ. Targeting RNA to treat neuromuscular disease. Nat Rev Drug Discov 2011;10(8):621-37.

[91] Hamed SA. Drug evaluation: PTC-124: a potential treatment of cystic fibrosis and Duchenne muscular dystrophy. IDrugs 2006;9(11): 783-9.

[92] Cirak S, Arechavala-Gomeza V, Guglieri M, et al. Exon skipping and dystrophin restoration in patients with Duchenne muscular dystrophy after systemic phosphorodiamidate morpholino oligomer treatment: an open-label, phase 2, dose-escalation study. Lancet 2011;378(9791):595-605.

[93] Goemans NM, Tulinius M, van den Akker JT, et al. Systemic administration of PRO051 in Duchenne muscular dystrophy. N Engl J Med 2011;364(16):1513-22.

[94] Oetting WS. Exome and genome analysis as a tool for disease identification and treatment: the 2011 Human Genome Variation Society scientific meeting. Hum Mutat 2012;33(3):586-90.

[95] Mendell JR, Shilling C, Leslie ND, et al. Evidence-based path to newborn screening for Duchenne muscular dystrophy. Ann Neurol 2012;71(3):304-13.

[96] Ferlini A. Neuromuscular disease: muscular dystrophy-something new on God's green earth?. Nat Rev Neurol 2012, Doi: 10.1038. 